\title{
Optimization of the force and power consumption of a microfabricated magnetic actuator
}

\author{
Juan José Zárate ${ }^{\mathrm{a}, *, 1}$, Giordano Tosolini ${ }^{\mathrm{b}, 1}$, Simona Petroni ${ }^{\mathrm{b}}$, Massimo De Vittorio ${ }^{\mathrm{b}, \mathrm{c}}$, \\ Herbert Shea ${ }^{a, * *}$ \\ a LMTS, Ecole Polytechnique Fédérale de Lausanne (EPFL), Neuchâtel 2002, Switzerland \\ b Center for Biomolecular Nanotechnologies UNILE, Istituto Italiano di Tecnologia, Via Barsanti, Arnesano, 73010 Lecce, Italy \\ c Dip. Ingegneria dell'Innovazione, Università del Salento, via per Monteroni, 73100 Lecce, Italy
}

\section{A R T I C L E I N F O}

\section{Article history:}

Received 16 February 2015

Received in revised form 12 August 2015

Accepted 12 August 2015

Available online 21 August 2015

\section{Keywords:}

Planar coil

Permanent magnet

Magnetic actuator

Power consumption

Tactile display

Haptic technology

\begin{abstract}
A B S T R A C T
The force $(F)$ and the power consumption $(P)$ of a magnetic actuator are modeled, measured and optimized in the context of developing micro-actuators for large arrays, such as in portable tactile displays for the visually impaired. We present a novel analytical approach complemented with finite element simulation (FEM) and experiment validation, showing that the optimization process can be performed considering a single figure of merit $F / \sqrt{P}$. The magnetic actuator is a disc-shaped permanent magnet displaced by planar microcoil. Numerous design parameters are evaluated, including the width and separation of the coil traces, the trace thickness, number of turns and the maximum and minimum radius of the coil. We obtained experimental values of $F / \sqrt{P}$ ranging from 2 to $12 \mathrm{mN} / \sqrt{\mathrm{W}}$ using up to 2-layer coils of both microfabricated and commercial printed circuit board (PCB) technologies. This performance can be further improved by a factor of two by adopting a 6-layer technology. The method can be applied to a wide range of electromagnetic actuators.
\end{abstract}

(C) 2015 Elsevier B.V. All rights reserved.

\section{Introduction}

Electromagnetic-based microactuators combine both high noncontact forces and large actuation strokes [1]. By using permanent magnets, high energy densities can be achieved. Such devices have been demonstrated for a wide range of applications, including labon-a-chip valves arrays [2], micromirrors arrays [3] and tactile displays $[4,5]$. While much research has focused on single microdevices or small arrays of magnetic actuators, electromagnetic (EM) actuation scales well to very large arrays of microdevices by using arrays of microfabricated planar coils driving arrays of small permanent magnets.

Haptic interfaces are central to assistive devices for blind and visually impaired people. Tactile displays generally consist of an array of independent vertical actuators, called taxels (the haptic counterparts of pixels). Several psychophysics strategies have been

\footnotetext{
* Corresponding author.

** Principal corresponding author at: rue de la Maladiere 71b, CP526, CH-2002 Neuchâtel, Switzerland.

E-mail addresses: juan.zarate@epfl.ch (J.J. Zárate), herbert.shea@epfl.ch (H. Shea).

1 These authors contributed equally to this work.
}

explored to provide graphical information through tactile displays, using static stimuli (shapes), vibrational stimuli (textures), or both $[6,7]$. Meanwhile, advances in the use of different actuation technologies in haptic devices have been recently reviewed [8,9], including the use of piezoelectric, electromagnetic, pneumatic and shape memory alloys. EM-based devices continue to be a promising option due to their bandwidth, scaling and portability characteristics. Several EM-based tactile display prototypes have been reported using 3D wire-wound inductors or similar technologies to attract or repel a small permanent magnet. The focus has been on small arrays (less than 100 taxels) generating vibration stimuli (from 20 to $200 \mathrm{~Hz}$ ) rather than static taxel displacement $[4,10,11$ ]. For those dynamic devices, actuation forces of $13 \mathrm{mN}$ using $400 \mathrm{~mW}$ power per taxel [4] and $9 \mathrm{mN}$ at $100 \mathrm{~mW}$ per taxel [10] were reported, with positive results on psychophysics tests. However, to display maps or other complex graphical information, larger tactile areas are needed (thousands of taxels, covering the area of a page).

Scaling up EM tactile displays to thousands of taxels and to areas of order $20 \mathrm{~cm} \times 20 \mathrm{~cm}$ adds important challenges for integration and power consumption. From an integration perspective, going to thousands to taxels implies an array of planar coils, as wire-wound solutions with high-permeability magnetic cores are not manufacturable for such high taxels count. From a power consumption and heat dissipation perspective for a portable device, the 
average power per taxel must be well below $100 \mathrm{~mW}$. The goal of this paper is to provide a model to allow optimizing an EM taxel for large arrays of mm-scale taxels.

In the framework of the Blindpad project, ${ }^{2}$ we are developing a tablet-sized 3000 taxel portable tactile display. Pitch size has been set in the range from 4 to $8 \mathrm{~mm}$. In 2004, Hale et al. recommended a threshold pressure for stimuli in fingertips of $60 \mathrm{mN} / \mathrm{cm}^{2}$, regardless the working taxel frequency [6]. However, more recent works have reported that the perception force threshold varies according to working frequency, sensing area, and surface shape [12-16]. Taking into account a pin diameter pin of $2.5 \mathrm{~mm}$ for our device, the required force should be of $3 \mathrm{mN}$ or higher, or $10 \mathrm{mN}$ for a pin with a $4 \mathrm{~mm} \times 4 \mathrm{~mm}$ area. Considering a typical $100 \mathrm{Wh}$ laptop battery and a proposed autonomy of $2.5 \mathrm{~h}$, the available average power is $40 \mathrm{~mW} /$ taxel. Power consumption can be also improved by incorporating a latching system that holds both taxel states, up and down. In this case, the coil is actuated only for switching, and there is no power consumption as long as the displayed image is not refreshed. Both force and power consumptions are key design drivers, as is scalability and integration aspects.

Analytical models to optimize the performance of coils or magnets for magnetic actuators with no presence of ferromagnetic materials are available in literature [5,17-19]. If the actuator does include a ferromagnetic material, the optimization could be better performed by using iterative algorithms like genetic algorithms $[20,21]$. These systems are much more complex to solved because of the non-linearity of the equations. However, none of the referred works present a simultaneous optimization of the power consumption and the magnetic force generated by a planar coil on a movable permanent magnet. For example, Fuestel et al. presented in [17] a polynomial analysis of the magnetic force as function of some of the design parameters, but using a fixed value of power. Kruussing presented in $[19,22]$ a study focused on the magnetic domains orientation and its influence in the force calculation, but without extending the analysis to the influence of the applied power.

Given our goal of a portable large-area tactile display based on EM microactuator, here we present the optimization of a magnetic microactuator, consisting of a planar microcoil and a magnetic disc suspended over the center of the coil, with no other magnetic elements. The taxel that is modeled was designed to be readily scalable to large arrays. A novel analytical approach was implemented, complemented with finite element simulation (FEM) and experimental studies. The influence of all key design parameters is analyzed in terms of force and power, comparing: analytical calculations, FEM simulations and measurements. Planar coils were fabricated in different designs using two fabrication technologies, and the magnetic force and power consumption were measured and compared with our model.

As a result of this analysis, a planar coil design is proposed to achieve the taxel requirements for a large array. The method can be applied to a wide range of electromagnetic actuators involving permanent magnets and planar coils, whenever a reduction of the power consumption is required, e.g. valves arrays [2] and micromirrors arrays [3].

\section{Magnetic system modeling}

The vertical actuator considered in this work consists of a discshaped permanent magnet bonded to an elastomer membrane and centered over a planar coil, as represented schematically in Fig. 1. When an electrical current is applied, the magnet is vertically attracted or repulsed due to the interaction with the induced

2 https://www.blindpad.eu

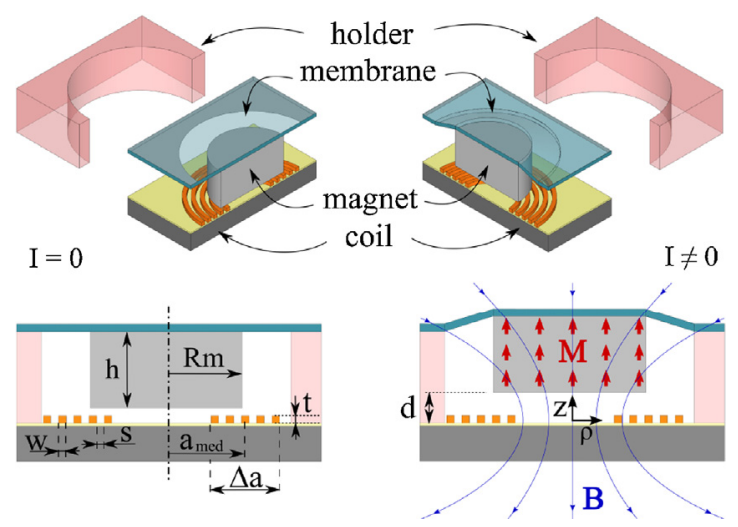

Fig. 1. Schematic of the magnetic system. A permanent magnet is suspended by an elastomer film over a planar coil, being attracted or repulsed when a current is passed through the coil. The parameters under analysis are indicated in the diagram: the magnet radius $R m$ and height $h$, the coil external dimensions $a_{\text {med }}$ and $\Delta a$ and the individual trace width $w$, separation $s$ and thickness $t$.

magnetic field. In this case it is possible to describe the system behavior by separately modeling the magnetic interaction between the magnet and the coil on one hand, and the elastic deformation of the elastomer membrane on the other.

The focus of the model presented in this section is to provide an analytical tool to precisely estimate the magnetic force $F$ between the magnet and the coil and the power consumption $P$. Given the size of the magnet, this model can be used to design the coil that maximize the actuator performance tacking into consideration the figure of merit $F / \sqrt{P}$. As pointed out later on, this ratio is independent of the electrical current and it allows to compare and optimize different coil designs. Being the actuation force and power the more challenging taxel constrains, the restoring force and the deflection of the membrane can be then tuned to a desired value by choosing the diameter, thickness and elastic properties of the elastomer [18].

Let us consider a permanent magnet of magnetization $\boldsymbol{M}$ and volume $V$, and a differential volume inside it with magnetic moment $\boldsymbol{m}=\boldsymbol{M} d V$. The force $\boldsymbol{f}$ acting on $\boldsymbol{M}$ due to an external magnetic field $\boldsymbol{B}$ can be expressed as [23],

$\boldsymbol{f}=(\boldsymbol{m} \cdot \nabla) \boldsymbol{B}=\nabla(\boldsymbol{m} \cdot \boldsymbol{B})$

The second form of Eq. (1) is valid for regions where $\nabla \times \boldsymbol{B}=0$, i.e. no electrical currents or time-dependent electrical fields are present, which is the case inside the magnetic volume. The total magnetic force is obtained by integrating Eq. (1) over the magnet volume,

$F_{i}=\sum_{V} f_{i}=\int_{V} \partial_{i}(\boldsymbol{M} \cdot \boldsymbol{B}(\boldsymbol{r})) d V$

where $I$ depends on the chosen coordinates system. Now using the divergence theorem, Eq. (2) can be transformed into a surface integral,

$F_{i}=\oint_{S}(\boldsymbol{M} \cdot \boldsymbol{B}(\boldsymbol{r}))(\hat{i} \cdot \hat{n}) d S$

The permanent magnet we consider is homogeneously and vertically magnetized $(\boldsymbol{M}=M \hat{z})$. Then by symmetry, no lateral force is expected if the magnet is located concentric with the planar coil. Only the vertical component of the force remains and can be calculated from Eq. (3),

$F_{z}=M\left[\int_{\text {top }} B_{z}(\boldsymbol{r}) d S-\int_{\text {bottom }} B_{z}(\boldsymbol{r}) d S\right]$

with the two surface integrals calculated over the top and bottom surfaces of permanent magnet. 
The magnetic field in a certain point $\boldsymbol{r}$ can be calculated by summing up the contribution of each $j$-turn of the coil,

$B_{z}(\boldsymbol{r})=\sum_{j=1}^{N} B_{z j}\left(I, a_{j}, \boldsymbol{r}\right)$

where $I$ is the current flowing through each of them. A simplification in the computation of the magnetic field is to consider each spiral turn as concentric one-dimensional conductive ring. In this case $a_{j}$ corresponds to the average radius.

Finally, the vertical component of magnetic field generated by one circular ring can be analytically calculated [19]:

$B_{z j}\left(I, a_{j}, \boldsymbol{r}\right)=\frac{\mu_{0} I}{2 a_{j}}\left[\frac{E\left(k_{\tilde{\rho}, \tilde{z}}\right) \frac{1-\tilde{\rho}^{2}-\tilde{z}^{2}}{(1-\tilde{\rho})^{2}+\tilde{z}^{2}}+K\left(k_{\tilde{\rho}, \tilde{z}}\right)}{\pi \sqrt{(1+\tilde{\rho})^{2}+(\tilde{z})^{2}}}\right]$

where the position vector $\boldsymbol{r}=\rho \hat{\rho}+z \hat{z}$ has been expressed in the normalized polar coordinates $\tilde{\rho}=\rho / a_{j}$ and $\tilde{z}=z / a_{j}$. The functions $E\left(k_{\tilde{\rho}, \tilde{z}}\right)$ and $K\left(k_{\tilde{\rho}, \tilde{z}}\right)$ are elliptical integrals of,

$k_{\tilde{\rho}, \tilde{z}}=\sqrt{\frac{4 \tilde{\rho}}{(1+\tilde{\rho})^{2}+\tilde{z}^{2}}}$.

The applied electrical power can be calculated directly by knowing the electrical resistance of the coil,

$P=R_{0} I^{2}=\frac{2 \pi \rho_{0} a_{m e d} N}{w t} I^{2}$

where $\rho_{0}$ is the electrical conductivity of the coil trace, $a_{m e d}$ the average radius of the $N$ turns and $(w t)$ is the cross section area of the trace (Fig. 1).

If the applied power is high enough, heating will cause the coil to increase its resistance. This effect can be considered in the power calculation by including the thermal factor,

$P=P_{0}(1+\alpha \Delta T)$

where $P_{0}$ is the non-thermal dependent power calculated in Eq. (8) and $\alpha$ is the linear temperature coefficient of electrical resistivity. The temperature increase $(\Delta T)$ can be expressed as the ratio between the average incoming power $(\bar{P})$ and the thermal conductance of the coil with the surroundings $(G)$. In the case of a latching actuation, the current is turned on only for a small fraction of the time. This fraction is given by the relation between the switching time $\left(\tau_{s}\right)$ and the average refresh time $\left(\tau_{r}\right)$. The average power is then calculated as $\bar{P}=P \tau_{s} / \tau_{r}$. For continuous current (CC) operation the particular case $\tau_{s}=\tau_{r}$ applies. The previous thermal considerations can be included in Eq. (9) as,

$P=P_{0}\left(\frac{1}{1-\overline{P_{0}} \alpha / G}\right)$

where $\overline{P_{0}}=P_{0} \tau_{s} / \tau_{r}$ is the non-thermal dependent average power. The factor in parentheses in Eq. (10) approaches 1 for $\overline{P_{0}} \ll G / \alpha$, and the power calculation of Eq. (8) is recovered. The ratio $G / \alpha$ has units of Watts and expresses from which value of average power the thermal dependence of resistivity needs to be taken into account. As discussed in Section 4.6, the values of average power per taxel considered for our portable application are small enough to neglect this correction.

The force depends linearly on the current (Eqs. (4)-(6)); while the power increases quadratically with the applied current (Eq. (8)). Since $F \propto I$ and $P \propto I^{2}$, the figure of merit $F / \sqrt{P}$ allows analyzing and comparing different actuator configurations independently of the applied current, which can be set later to reach the desired force.

Magnetic force and electrical power calculations were implemented using a $\mathrm{C}$ routine and the GSL library for the elliptical
Table 1

Summary of fabricated coils used in this work. The first eight rows correspond to microfabricated coils, while the last one is based on PCB technology.

\begin{tabular}{lllcc}
\hline Name & $N$ & $\begin{array}{l}m=w /(w+s) \\
\pm 9 \%\end{array}$ & $\begin{array}{c}\Delta a[\mu \mathrm{m}] \\
(\text { nominal) }\end{array}$ & $\begin{array}{c}R_{\text {tot }}[\Omega] \\
\pm 1 \%\end{array}$ \\
\hline$\mu$ Fab-A & 23 & 0.63 & 715 & 96 \\
$\mu$ Fab-B & 20 & 0.54 & 725 & 89 \\
$\mu$ Fab-C & 14 & 0.38 & 725 & 87 \\
$\mu$ Fab-D & 28 & 0.38 & 1425 & 157 \\
$\mu$ Fab-E & 20 & 0.38 & 1025 & 104 \\
$\mu$ Fab-F & 24 & 0.38 & 1225 & 128 \\
$\mu$ Fab-G & 8 & 0.38 & 425 & 46 \\
$\mu$ Fab-A-2L & $2 \times 23$ & 0.63 & 425 & 230 \\
$P C B-2 L$ & $2 \times 4$ & 0.50 & 1350 & 0.2 \\
\hline
\end{tabular}

integrals [24]. Finite Element Simulations (FEM) were used as a first validation of the analytical model, using the commercial software Comsol Multiphysics. In view of the symmetry of the system, the 2D axisymmetric configuration was used, assuming an homogeneous vertical magnetization in the magnet region and constant current distribution over the turns path.

\section{Coil fabrication and experimental setup}

In order to experimentally validate the analytical model, we fabricated several designs of planar microcoils. The generic structure of the coil consists of a multi-turn, with one or two level metal layer spiral. The parameters $w$ and $s$ vary from $10 \mu \mathrm{m}$ to $50 \mu \mathrm{m}$ while the ratio $a_{\min } / a_{\max }$ varies between $300 / 1700 \mu \mathrm{m}$ and $800 / 1200 \mu \mathrm{m}$ (i.e. $N$ varies from 8 to 35). The analyzed designs are summarized in Table 1.

The coils were fabricated on a standard $\langle 100\rangle$ p-type silicon wafer with a $1 \mu \mathrm{m}$ thick thermal oxide. As first process step, a $2 \mu \mathrm{m}$ of $99.99 \%$ aluminum layer was sputtered, followed by the first photolithography. The bottom electrode or the first coil level was defined via $\mathrm{SiCl}_{4} / \mathrm{Ar} / \mathrm{N}_{2}$ inductive coupled plasma reactive ion etching (ICP RIE) [25]. A $2 \mu \mathrm{m}$ thick silicon oxide layer was then sputtered to insulate the first metal layer. After performing the second photolithography, the via holes were opened into the $\mathrm{SiO}_{2}$ by buffered oxide etch (BOE). The second layer of aluminum was deposited via sputtering and defined by dry etching. In Fig. 2, we
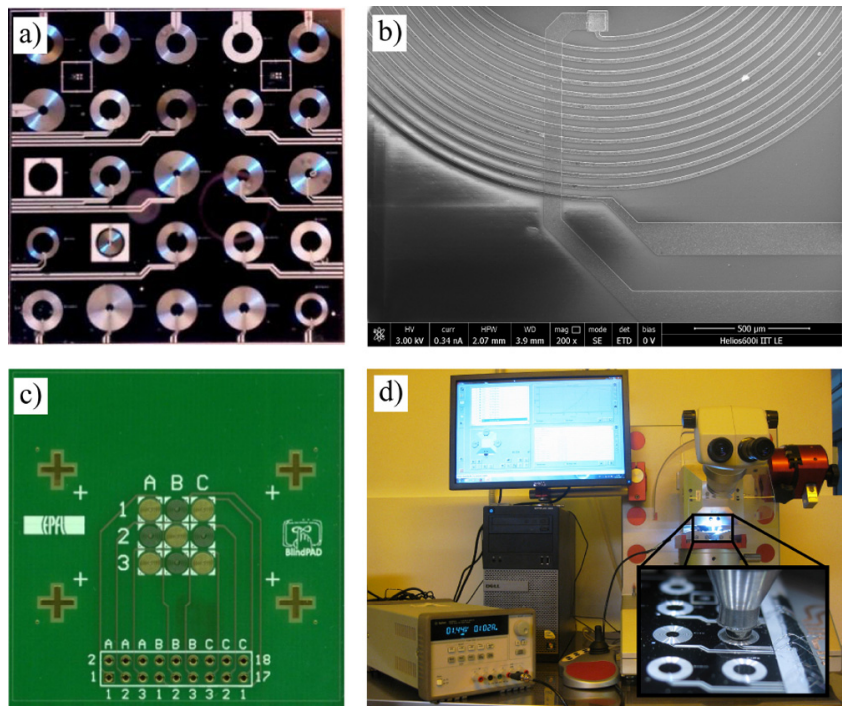

Fig. 2. Images of the fabricated coils and the experimental setup. (a) Optical image of the microfabricated die with different planar coil designs. (b) SEM image of one microfabricated coil, the result of the double layer process can be observed. (c) Top view of a $3 \times 3$ array of the same actuators, implemented in a commercial PCB technology. (d) Experimental setup for the force-displacement measurements. 
report an optical image of a die with the different coil designs (Fig. 2a) and a SEM micrograph of a coil detail at the end of the fabrication process (Fig. 2b).

In order to test the system in an array, we produced also a $3 \times 3$ array of coils with the same design. For this configuration we used standard printed circuit board technology (PCB) with wider $w$ and $s$ dimensions (Fig. 2c). A 2-layer PCB technology was chosen, with $35 \mu \mathrm{m}$ thick $\mathrm{Cu}$ layers separated by $1 \mathrm{~mm}$.

The planar coils were experimentally tested to obtain values of magnetic force and power dissipation as function of the design parameters. The electrical characteristics of the conductors were measured using a manual probe station Karl Süss PM5 and a semiconductor parameter analyzer Agilent 4155 with a 4-point probes method. The $I-V$ curves were acquired by sweeping the current between $-0.1 \mathrm{~A}$ and $+0.1 \mathrm{~A}$ while recording the voltage. An average value of electrical resistivity $\rho_{A l}=(3.3 \pm 0.1) \times 10^{-8} \Omega \mathrm{m}$ was obtained for the aluminum coil traces.

The interaction force measurement setup is presented in Fig. 2d. A magnetic disc $\left(R_{m}=2 \mathrm{~mm}, h=1 \mathrm{~mm}, N 48\right.$ grade and $B_{r}=1.37-1.42 \mathrm{~T}$, from Supermagnete) was attached to the probe of a pull shear tester Condor EZ from XYZTEC. A power supply E3631 A from Agilent was used to bias the coils. The force was recorded continuously while approaching the magnet to the coil from a distance of $1 \mathrm{~mm}$ to $0.1 \mathrm{~mm}$.

\section{Results and discussion}

We first evaluated the force dependence on the magnet-coil distance. The inset in Fig. 3 shows the experimental data of $F$ vs $d$ for three different values of current on the representative sample $\mu$ Fab-F. As expected, the interaction force increases as the magnet gets closer to the coil and if the applied current is increased. To compare this experimental results with the analytical model and the FEM simulations, the force data are normalized by the squared root of the applied power and plotted in Fig. 3. The three experimental groups of data $(I=50,100$ and $150 \mathrm{~mA})$ collapse on the same curve and agree very well with the analytical curve and FEM simulations.

The $F / \sqrt{P}$ information in Fig. 3 is significant because allows calculating the generated force at any applied power. For example, with the obtained value $F / \sqrt{P}=2.3 \mathrm{mN} / \sqrt{\mathrm{W}}$ (i.e. $d=0.2 \mathrm{~mm}$ in Fig. 3), the system can provide a force of $2.3 \mathrm{mN}$ at $1 \mathrm{~W}$; or $0.7 \mathrm{mN}$ at $0.1 \mathrm{~W}$ and so forth. The current to drive the coil at the desired power can be calculated by knowing the resistance, using $I=\sqrt{P / R}$.

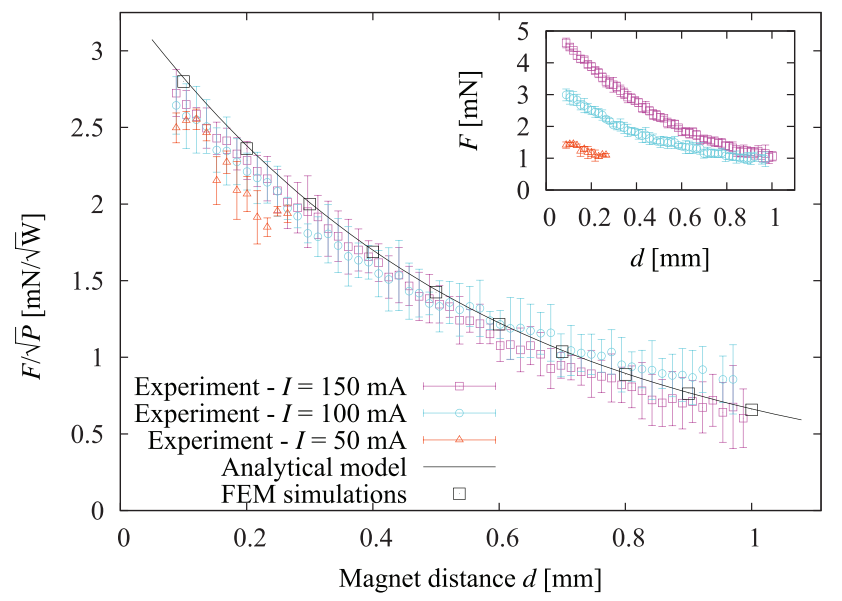

Fig. 3. Measured and computed figure of merit $F / \sqrt{P}$ as a function of the magnet distance $d$, for the sample $\mu$ Fab-F. All the measured points collapse to a single curve, in excellent agreement with the analytical curve and FEM simulations points. Inset: Measured values of $F$ as function of the magnetic distance $d$, for values of applied current $I=50,100$ and $150 \mathrm{~mA}$.

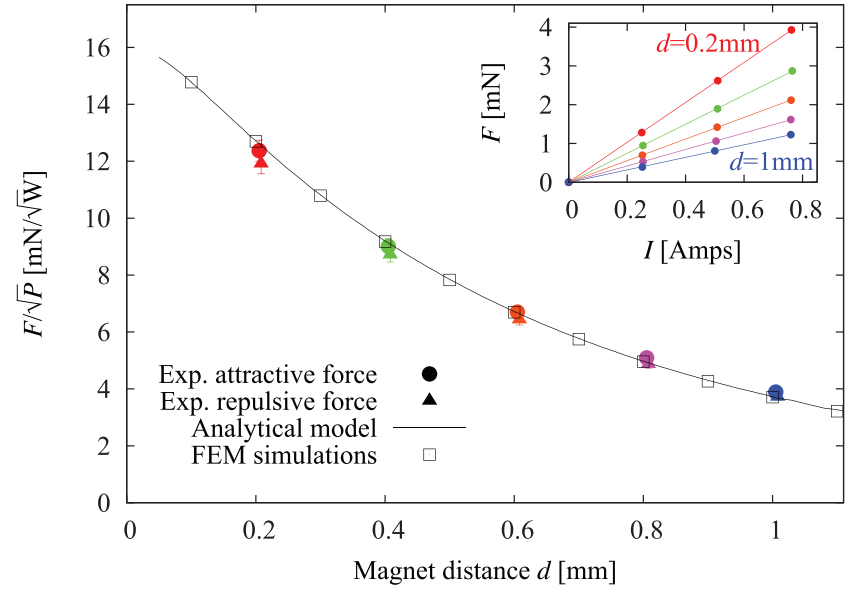

Fig. 4. Measured and computed figure of merit $F / \sqrt{P}$ as a function of the magnet distance $d$, for the sample PCB-2L. As in Fig. 3, the agreement between the experimental, analytical and FEM simulation results are excellent. Inset: Measured $F$ vs $I$ for values of $d=0.2,0.4,0.6,0.8$ and $1 \mathrm{~mm}$. The slope of each curves is used to obtain the experimental value of $F / \sqrt{P}$.

In subsequent analyses and in order to obtain accurate experimental results of $F / \sqrt{P}$, the linear fit $F=a \cdot I$ has been used (inset in Fig. 4). The slopes $a$ are multiplied by $1 / \sqrt{R}$ to calculate the experimental points of $F / \sqrt{P}$ in the main graph of Fig. 4. In this graph, both attractive and repulsive forces were studied by switching the current direction.

In the following subsections the optimization process is described. First the magnet dimensions and magnetization values are analyzed. Then the coil design parameters are examined. The parameters $w$ and $s$ are analyzed taking into consideration the metallization ratio $m=w /(w+s)$, and studied together with the number of turns $N$. Finally the influence of the coil external dimensions is investigated, in terms of $a_{\text {med }}, \Delta a$ and $t$. In all the cases the actuator performance is evaluated in terms of $F / \sqrt{P}$, comparing the experimental results with the analytical model and FEM simulations.

\subsection{Magnet dimensions and magnetization}

As expected from Eq. (2), the force increases with magnetization value and with the magnet volume, if the coil is proportionally scaled up. To be compatible with the minimum taxel dimension ( $4 \mathrm{~mm} \times 4 \mathrm{~mm}$ ), the magnet radius was fixed to $R m=1 \mathrm{~mm}$, to allow an annular space for the holder placement and the restoring membrane deformation (Fig. 1). In the analysis the magnet height was also fixed to $h=1 \mathrm{~mm}$, region where the magnetic field gradient $\nabla$ $\boldsymbol{B}$ is stronger.

For higher force and energy density, neodymium permanent magnets are generally selected because of their outstanding magnetic properties [26]. From a microfabrication and ease of assembly perspective, the use of magnetic materials that can be directly deposited in a batch fabrication process might be appealing. However, these materials have much lower permanent magnetization and they hence are not considered in this work. For permanent magnets the magnetization value $m$ can be obtained from the remanent field $\left(B_{r}\right)$ as $M=B_{r} / \mu_{0}$. However, this calculation of $m$ is accurate only when the magnet is in a closed ferromagnetic loop. When this condition is not satisfied, the average magnetization value can be lower due to demagnetization effects $[27,28]$. In view of this, we used a value $M=0.9 B_{r} / \mu_{0}=1.25 \mathrm{~T} / \mu_{0}$ for the analytical model and FEM calculations, which agrees with similar previous research [17]. 


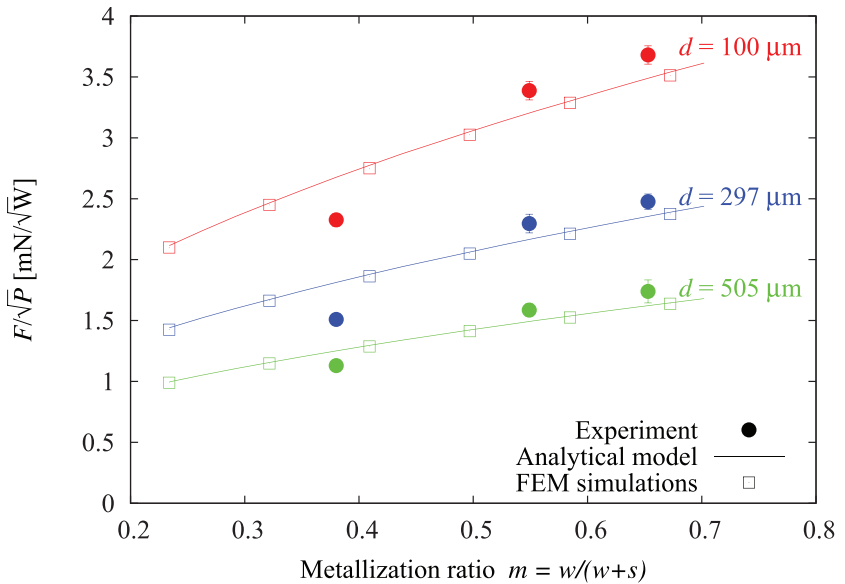

Fig. 5. Figure of merit $F / \sqrt{P}$ as a function of the metallization ratio $m$, for three different values of magnet distance $d$. The experimental points correspond to three different microfabricated samples. Increasing $m$ reduces the consumption of power for the same applied current, and therefore the device performance is improved.

\subsection{Metallization ratio and number of turns}

The actuator performance improves with increasing metallization ratio $m$, keeping other parameters fixed, as plotted in Fig. 5. This is due to the decrease of power consumption when the conductor cross section is increased.

The figure of merit $F / \sqrt{P}$ is roughly independent on the number of turns $N$, as long as other parameters like $\Delta a, a_{\text {med }}, m$ and $t$ remain constant. On one hand the coil-generated magnetic force scales up with the product $(N \times I)$ in Eqs. (5) and (7). On the other hand, by replacing $w=m \Delta a /(N-1)$ in Eq. (8), it can be shown that the applied power approximately scales with $(N \times I)^{2}$.

\subsection{Coil lateral dimensions}

The lateral dimensions of the coil can be fully defined by two variables: mean radius, $a_{\text {med }}$, and total coil width, $\Delta a$. We fixed $a_{m e d}=R m$ since this optimizes the force for a given current, as the one-turn loop (with $a_{j}=R m$ in Eq. (5)) has the higher contribution to $F / \sqrt{P}$. Fig. 6 shows the dependence of $F / \sqrt{P}$ against $\Delta a$. On one hand, for low $\Delta a$ values, the actuator performance is decreased because of the higher current density. On the other hand, at high

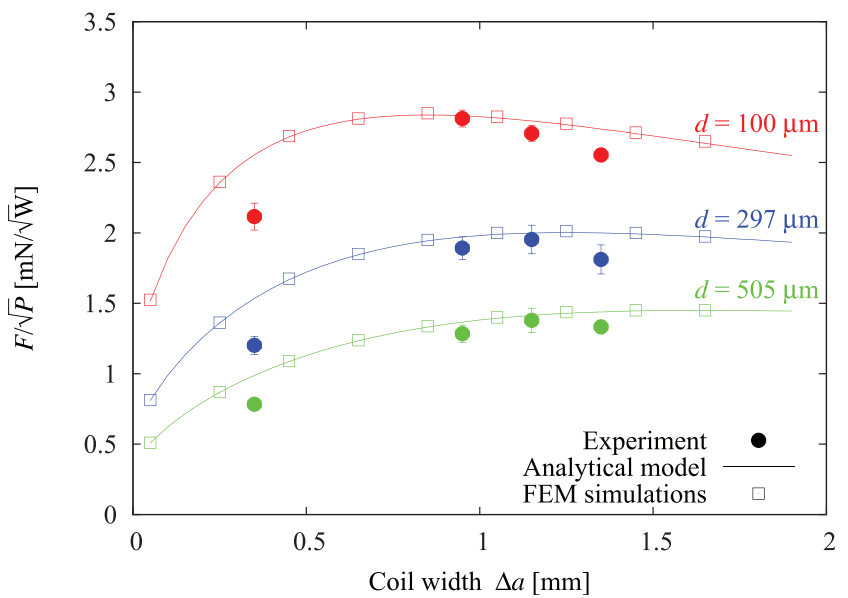

Fig. 6. Figure of merit $F / \sqrt{P}$ as a function of the coil width $\Delta a$, for three different values of magnet distance $d$. The experimental points correspond to four different microfabricated samples. For small values of $\Delta a$, the current density is higher and so the power consumption, too. In the opposite limit, sufficiently large values of $\Delta a$ generate a less effective magnetic field gradient, and therefore the force decreases.
$\Delta a$ values, a wider coil makes the increase of power consumption higher than the improvement of the force. An optimal value coil width was found to be around $\Delta a \simeq 1.2 \mathrm{~mm}$. Moreover, this maximum is weakly dependent on the magnet distance $d$.

\subsection{Coil thickness}

By increasing the conductor thickness $t$ (Eq. (8)), the power consumption decreases, while the force remains the same, so the ratio $F / \sqrt{P}$ is enhanced. That is valid for $t \ll d$. When $t$ becomes comparable to $d$, the force depends on $t$ and increasing the coil thickness is not effective to improve the figure of merit. Anyhow, the maximum conductor thickness is limited by the maximum aspect ratio $w / t$ possible for the coil fabrication technologies.

Multilayer techniques can be used, increasing the total coil volume available while keeping reasonable fabrication aspect ratios and resistance values. Different conductive thickness samples are compared in Fig. 7. $\mu$ Fab-A points correspond to microfabricated samples with one and two Al conductive layers, while the $P C B$ $2 \mathrm{~L}$ point corresponds to the double $\mathrm{Cu}$-layer coil implemented in standard PCB technology. In order to compare these experimental data with a several layer coil, we plotted a third series of analytical obtained points for a typical 6-layer PCB technology with thicknesses of $25 \mu \mathrm{m}$ and $50 \mu \mathrm{m}$ for the metal and the dielectric layers, respectively. In Fig. 7 one can see that the $F / \sqrt{P}$ figure of merit is greatly improved in the case of six layers, almost a factor two compared with the measured PCB sample.

\subsection{Drive electronics considerations}

Driving a single taxel in the lab is straightforward, but the portability of the tactile display imposes constraints on the drive electronic to push current through thousands of independent coils while consuming less than $100 \mathrm{~W}$ and mass of under $1 \mathrm{~kg}$. The parameters $N, w$ and $s$ should be tuned to obtain a coil resistance higher than $R_{\text {lim }}$, with the limit value $R_{\text {lim }} \simeq 1 \Omega$ given by the constraint of scalable drive electronics. For lower resistance values the drive electronics for an array of a thousand of coils become much more challenging. The inductance $L$ for all the coils considered in this work were in the range between 0.5 and $5 \mu \mathrm{H}$. These inductive loads can become relevant in an AC operation, such as a vibration stimuli. However for the proposed DC taxel

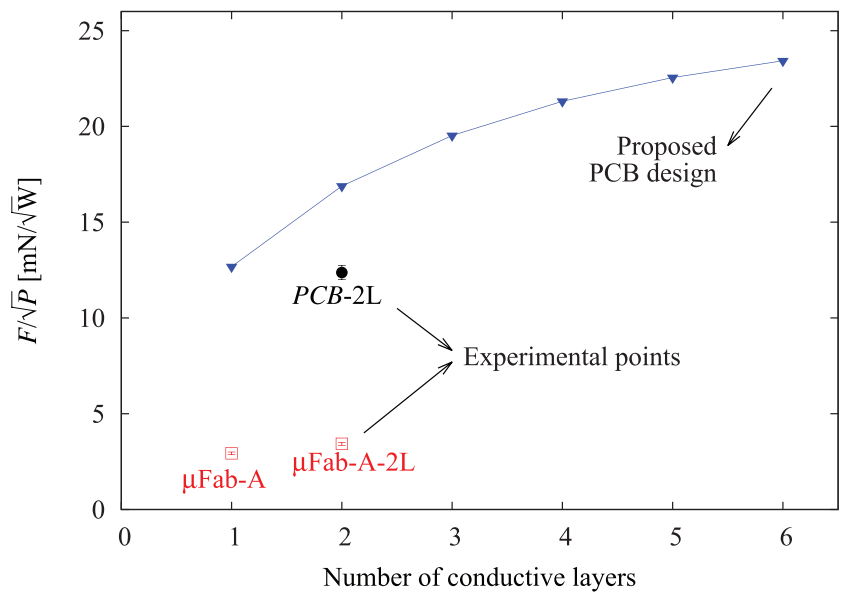

Fig. 7. Figure of merit $F / \sqrt{P}$ as a function of the number of conductive layers. The experimental results from samples $\mu$ Fab-A, $\mu$ Fab-A-2L and $P C B-2 \mathrm{~L}$ are plotted. The analytical predictions for an up to 6 -layer commercial PCB process are also included. In the PCB, $25 \mu \mathrm{m}$ thick conductive layers are considered, isolated by $50 \mu \mathrm{m}$ thick dielectric layers. 
operation, considering $L=5 \mu \mathrm{H}$ and an actuation ramp of $1 \mathrm{~A} / \mathrm{ms}$, an induced voltage of $5 \mathrm{mV}$ is expected, which can easily be managed.

\subsection{Thermal effects in the coil}

The influence of Joule heating in the coils was considered. Thermal conductivity $G$ was obtained for the different designs by Comsol simulations and analytical calculations. In Eq. (10), higher values of $G$ lead to lower thermal corrections. In all cases $G$ was underestimated to obtain the most conservative analysis by only considering the thermal conduction through a heat sink below the coil. The lowest thermal conductivity was found for sample $P C B-2 L$, with $G=6 \times 10^{-3}[\mathrm{~W} / \mathrm{K}]$. The values of $G$ obtained for the other samples were $15 \times 10^{-3}[\mathrm{~W} / \mathrm{K}]$ for the 6 -layers coil and $\sim 1[\mathrm{~W} / \mathrm{K}]$ the microfabricated samples.

For sample $P C B-2 L$, using the thermal resistivity of copper $\left(\alpha=3.8 \times 10^{-3}[1 / \mathrm{K}]\right)$, the ratio $G / \alpha$ is $1.6[\mathrm{~W}]$. For an average applied power of $40 \mathrm{~mW}$, the value $P=1.026 P_{0}$ is obtained (Eq. (10)). This small difference was neglected and Eq. (8) was used for the calculation of the power, where $P_{0}$ is simply proportional to $I^{2}$.

\subsection{Interaction between magnets}

The actuation force and power of each taxel can be calculated by considering only the magnet-coil interaction. However, in an array, the magnetic crosstalk between magnets could be high enough to destabilize the array of taxels. As examples, two orientation configurations were considered using arrays of $30 \times 30$ magnets: an all-north-up ( $\uparrow \uparrow)$ and an alternating $(\uparrow \downarrow \uparrow)$ arrangement. The magnetic field generated by each magnet on the array was computed as a dipole. We validated this approximation by comparing the magnetic field generated by a disc-shape magnet (calculated by Comsol FEM simulations) with the dipole magnetic field. At the distance of the first neighbor, the difference between the FEM calculations and the dipole model was less than $6 \%$. Three kinds of instability in the center of the array were study. (a) The central test magnet was moved in the array plane $\left(\Delta x_{0}\right)$ and the lateral force was calculated $\left(F x_{c t}\right)$. (b) It was moved up $\left(\Delta z_{0}\right)$ and the vertical force was computed $\left(F z_{c t}\right)$. (c) The test magnet was rotated in its original position $\left(\Delta \theta_{0}\right)$ and the generated torque was computed $\left(\tau y_{c t}\right)$.

The crosstalk calculations are reported in Table 2, for pitch sizes of 4,6 and $8 \mathrm{~mm}$. The negative sign in a force or a torque represents a restitutive interaction, while a positive sign means a non-restitutive interaction. In all the cases the alternating configuration reduces the absolute value of $F x_{c t}$ and $F z_{c t}$ by $35 \%$, and $\tau_{c t}$ by $70 \%$. Also, using the alternating array the two strongest crosstalk interactions, $F z_{c t}$ and $\tau_{c t}$, become a restitutive force and torque respectively. These two advantages make the alternating configuration the best choice in terms of magnetic crosstalk instability. A scaling behavior Pitch ${ }^{-n}$ is observed for the calculated forces $(n=4)$

Table 2

Magnetic crosstalk calculations for three different pitch sizes. The magnetic fields, forces and torques were calculated under the approximation of dipole-dipole interactions. The negative sign in a force or a torque represents a restitutive interaction. Two magnets orientation in the array were considered: an all-north-up ( $\uparrow \uparrow)$ and an alternating $(\uparrow \downarrow \uparrow)$ arrangement.

\begin{tabular}{|c|c|c|c|c|c|c|}
\hline \multirow[t]{2}{*}{ Pitch [mm] } & \multicolumn{2}{|c|}{$\begin{array}{l}\text { (a) } F x_{c t}[\mathrm{mN}] \\
\Delta x_{0}=0.2 \mathrm{~mm}\end{array}$} & \multicolumn{2}{|c|}{$\begin{array}{l}\text { (b) } F z_{c t}[\mathrm{mN}] \\
\Delta z_{0}=0.2 \mathrm{~mm}\end{array}$} & \multicolumn{2}{|c|}{$\begin{array}{l}\text { (c) } \tau_{c t}[\mathrm{mN} \mathrm{mm}] \\
\Delta \theta_{0}=10^{\circ}\end{array}$} \\
\hline & $\uparrow \uparrow \uparrow$ & $\uparrow \downarrow \uparrow$ & $\uparrow \uparrow \uparrow$ & $\uparrow \downarrow \uparrow$ & $\uparrow \uparrow \uparrow$ & $\uparrow \downarrow \uparrow$ \\
\hline 4 & -4.4 & 2.9 & 8.7 & -5.6 & 23 & -7 \\
\hline 6 & -0.58 & 0.38 & 1.15 & -0.74 & 6.8 & -2.1 \\
\hline 8 & -0.14 & 0.09 & 0.27 & -0.18 & 2.9 & -0.9 \\
\hline
\end{tabular}

Table 3

Summary of optimization process carried out for the $P C B$ - $2 L$ sample and for the next generation of 6-layer coil (see Fig. 7). The criteria of choice for each design parameter are pointed out, while the obtained results for both designs are showed at the four finals rows. Note: $\uparrow=$ as big as possible; $\Downarrow=$ as small as possible.

\begin{tabular}{llll}
\hline & $P C B-2 L$ & $P C B-6 L$ & Optimization \\
\hline$R m[\mathrm{~mm}]$ & 1 & 1 & $\uparrow$, taxel size \\
$h[\mathrm{~mm}]$ & 1 & 1 & Opt. to $\nabla B$ strong \\
$B_{r}[\mathrm{~T}]$ & 1.25 & 1.25 & $\uparrow$ \\
$d[\mu \mathrm{m}]$ & $50 \rightarrow 250$ & $50 \rightarrow 250$ & Taxel dynamic range \\
$s[\mu \mathrm{m}]$ & 150 & 150 & $\Downarrow$ \\
$w[\mu \mathrm{m}]$ & 150 & 150 & $\uparrow, R>R_{\text {lim }}$ \\
$a_{\text {med }}[\mathrm{mm}]$ & 1 & 1 & Optimized, $\simeq R m$ \\
$\Delta a[\mathrm{~mm}]$ & 0.9 & 1.2 & Optimized \\
$N$ & 4 & 11 & $=1+\Delta a /(w+s)$ \\
$N_{\text {layers }}$ & 2 & 6 & Optimized \\
$t_{\text {copper }}[\mu \mathrm{m}]$ & $2 \times 35$ & $6 \times 25$ & $\uparrow$ \\
$t_{\text {insulator }}[\mu \mathrm{m}]$ & $1 \times 1000$ & $5 \times 50$ & $\Downarrow$ \\
$F / \sqrt{P}[\mathrm{mN} / \sqrt{\mathrm{W}}]$ & 12 & 23 & Result, @d $=0.2 \mathrm{~mm}$ \\
$R[\Omega]$ & 0.2 & 5 & Result \\
$I(1 \mathrm{~W})[\mathrm{A}]$ & 2.2 & 0.44 & Result \\
$I(40 \mathrm{~mW})[\mathrm{A}]$ & 0.45 & 0.09 & Result \\
\hline
\end{tabular}

and torque $(n=3)$. This dependence with the separation between magnets is expected from a dipole-dipole interaction. Finally it can be observed that for a pitch size of $4 \mathrm{~mm}$ the crosstalk interactions are of the same order of the actuation forces required for finger stimulation (Section 1). However if the pitch size is increased to $6 \mathrm{~mm}$, the magnetic crosstalk is rapidly reduced by a factor of $1 / 8$.

\subsection{Taxel performance and further improvements}

As mentioned in Section 1, the threshold force for the fingerprint perception is generally between 3 and $10 \mathrm{mN}$ for a pin diameter between 2 and $4 \mathrm{~mm}$. For a portable application, power consumption should be at most $40 \mathrm{~mW} /$ taxel. Both specifications can be satisfied considering the design described in Section 4.4. A value of $F / \sqrt{P} \simeq 23 \mathrm{mN} / \sqrt{\mathrm{W}}$ was obtained for proposed 6 -layer coil. This corresponds to a force $F=4.6 \mathrm{mN}$ at $P=40 \mathrm{~mW}$ for a taxel operating in CC mode. Using a latching mechanism with a $1 \%$ of duty cycle, a force ten times higher is obtained for the same average power. Latching would also help to compensate for magnetic crosstalk with a $4 \mathrm{~mm}$ taxel pitch.

All the design parameters schematized in Fig. 1 have been optimized to obtain this performance. In Table 3 the optimization process is summarized, for the $P C B-2 L$ fabricated sample and for the proposed next 6-layer coil generation. For more complex geometries or with the use of other materials, the magnetic force can be further increased. By focusing the magnetic field with soft ferromagnetic materials, the interaction force can be improved and taxel-taxel magnetic crosstalk can be reduced. Adding soft magnetic material should be to aimed at improving the magnetic gradient $\nabla \boldsymbol{B}$, rather than the absolute value $\boldsymbol{B}$. In this case iterative non-linear algorithms can be used to compute and optimize the magnetic force.

\section{Conclusion}

With this work we provided a powerful analytical tool to optimize the magnetic actuation force between a magnet and a planar coil. The actuator consists in a disc-shaped permanent magnet centered over a planar microcoil. This electromagnetic technology allows scaling up to arrays of thousands of actuators for haptic applications. The analytical model has been validated by FEM simulation and by experimental results, using microfabricated and PCB coils. All the design parameters were analyzed using the figure of merit $F / \sqrt{P}$, allowing the analysis to be performed 
under the same power conditions. The optimal coil dimension was found for a given magnet size of $2 \mathrm{~mm}$ diameter, $1 \mathrm{~mm}$ height. A value of $F / \sqrt{P} \simeq 12 \mathrm{mN} / \sqrt{\mathrm{W}}$ was obtained for a standard PCB sample and a value of $F / \sqrt{P} \simeq 23 \mathrm{mN} / \sqrt{\mathrm{W}}$ is expected using a commercial available 6-layer PCB technology. The experimental value we obtained is at least $50 \%$ higher than values reported in the literature for similar devices based on planar coils $[4,17,29]$. We expect to double this performance in the next generation. With this last $F / \sqrt{P}$ value, an actuation force of $4.6 \mathrm{mN}$ at $40 \mathrm{~mW}$ can be achieved for a CC operation. Presented results are compatible with both, the psychophysical perception thresholds and the power consumption of a portable haptic tactile display device.

\section{Acknowledgements}

This work was supported by the EU within the BLINDPAD Project (FP7/2007-2013 Grant Agreement No. 611621) and by PONa3_00077 ITEM project.

\section{References}

[1] O. Cugat, J. Delamare, G. Reyne, Magnetic micro-actuators and systems (MAGMAS), IEEE Trans. Magn. 39 (6) (2003) 3607-3612, http://dx.doi.org/10.1109/ TMAG.2003.816763.

[2] P.J. Chang, F.W. Chang, M.C. Yuen, R. Otillar, D.A. Horsley, Force measurements of a magnetic micro actuator proposed for a microvalve array, J. Micromech. Microeng. 24 (3) (2014) 034005, http://dx.doi.org/10.1088/0960-1317/24/3/ 034005.

[3] Ç. Ataman, S. Lani, W. Noell, N. de Rooij, A dual-axis pointing mirror with moving-magnet actuation, J. Micromech. Microeng. 23 (2) (2013) 025002, http://dx.doi.org/10.1088/0960-1317/23/2/025002.

[4] M. Benali-Khoudja, M. Hafez, A. Kheddar, VITAL: an electromagnetic integrated tactile display, Displays 28 (3) (2007) 133-144, http://dx.doi.org/10.1016/j. displa.2007.04.013.

[5] J. Streque, A. Talbi, P. Pernod, V. Preobrazhensky, New magnetic microactuator design based on PDMS elastomer and MEMS technologies for tactile display, IEEE Trans. Haptics 3 (2) (2010) 88-97, http://dx.doi.org/10.1109/TOH.2009. 61.

[6] K. Hale, K. Stanney, Deriving haptic design guidelines from human physiological psychophysical and neurological foundations, IEEE Comput. Graph. Appl. 24 (2) (2004) 33-39, http://dx.doi.org/10.1109/MCG.2004.1274059.

[7] V. Chouvardas, A. Miliou, M. Hatalis, Tactile displays: overview and recent advances, Displays 29 (3) (2008) 185-194, http://dx.doi.org/10.1016/j.displa. 2007.07.003.

[8] H. Ishizuka, N. Miki, MEMS-based tactile displays, Displays 37 (2015) 25-32, http://dx.doi.org/10.1016/j.displa.2014.10.007.

[9] F. Vidal-Verdu, M. Hafez, Graphical tactile displays for visually-impaired people, IEEE Trans. Neural Syst. Rehabil. Eng. 15 (1) (2007) 119-130, http://dx.doi. org/10.1109/TNSRE.2007.891375.

[10] J. Streque, A. Talbi, P. Pernod, V. Preobrazhensky, Pulse-driven magnetostatic micro-actuator array based on ultrasoft elastomeric membranes for active surface applications, J. Micromech. Microeng. 22 (9) (2012) 095020, http://dx.doi. org/10.1088/0960-1317/22/9/095020.

[11] C. Bolzmacher, G. Chalubert, O. Brelaud, J.-P. Alexander, M. Hafez, Morphing tactile display for haptic interaction in vehicles, in: M. Auvray, C. Duriez (Eds.), Haptics: Neuroscience, Devices, Modeling, and Applications, Lecture Notes in Computer Science, Springer, Berlin, Heidelberg, 2014, pp. 333-341.

[12] H.S. Lee, H. Phung, D.-H. Lee, U.K. Kim, C.T. Nguyen, H. Moon, J.C. Koo, J.-d. Nam, H.R. Choi, Design analysis and fabrication of arrayed tactile display based on dielectric elastomer actuator, Sens. Actuators A: Phys. 205 (2014) 191-198, http://dx.doi.org/10.1016/j.sna.2013.11.009.

[13] J. Dosher, B. Hannaford, Human interaction with small haptic effects, Presence 14 (3) (2005) 329-344, http://dx.doi.org/10.1162/105474605323384672.

[14] H. King, R. Donlin, B. Hannaford, Perceptual thresholds for single vs. multifinger haptic interaction, in: 2010 IEEE Haptics Symposium, 2010, pp. 95-99, http://dx.doi.org/10.1109/HAPTIC.2010.5444670.

[15] S. Louw, A.M.L. Kappers, J.J. Koenderink, Active haptic detection and discrimination of shape, Percept. Psychophys. 64 (7) (2002) 1108-1119, http://dx.doi. org/10.3758/BF03194760.

[16] A.D. Berquin, V. Lijesevic, S. Blond, L. Plaghki, An adaptive procedure for routine measurement of light-touch sensitivity threshold, Muscle Nerve 42 (3) (2010) 328-338, http://dx.doi.org/10.1002/mus.21689.

[17] A. Feustel, O. Krusemark, J. Müller, Numerical simulation and optimization of planar electromagnetic actuators, Sens. Actuators A: Phys. 70 (3) (1998) 276-282, http://dx.doi.org/10.1016/S0924-4247(98)00103-4.

[18] D. de Bhailís, C. Murray, M. Duffy, J. Alderman, G. Kelly, S. Ó Mathúna, Modelling and analysis of a magnetic microactuator, Sens. Actuators A: Phys. 81 (1-3) (2000) 285-289, http://dx.doi.org/10.1016/S0924-4247(99)00176-4.
[19] A. Kruusing, Optimizing magnetization orientation of permanent magnets for maximal gradient force, J. Magn. Magn. Mater. 234 (3) (2001) 545-555, http:// dx.doi.org/10.1016/S0304-8853(01)00267-0.

[20] C.H. Ko, J.C. Chiou, Optimal design of the magnetic microactuator using the genetic algorithm, J. Magn. Magn. Mater. 263 (1) (2003) 38-46, http://dx.doi. org/10.1016/S0304-8853(02)01533-0.

[21] C. Pompermaier, F. Kalluf, M. Ferreira da Luz, N. Sadowski, Study and optimization of a small tubular linear motor with permanent magnet, in: 2010 XIX International Conference on Electrical Machines (ICEM), 2010, pp. 1-6, http:// dx.doi.org/10.1109/ICELMACH.2010.5608464.

[22] A. Kruusing, Actuators with permanent magnets having variable in space orientation of magnetization, Sens. Actuators A: Phys. 101 (1-2) (2002) 168-174, http://dx.doi.org/10.1016/S0924-4247(02)00154-1.

[23] J.D. Jackson, Classical Electrodynamics, Wiley, New York, 1962.

[24] M.E.A. Galassi, GNU Scientific Library Reference Manual, Network Theory Limited, Bristol, 2009.

[25] M. Amato, F. Dalena, C. Coviello, M. De Vittorio, S. Petroni, Modeling, fabrication and characterization of micro-coils as magnetic inductors for wireless power transfer, Microelectron. Eng. 111 (2013) 143-148, http://dx.doi.org/10.1016/j. mee.2013.03.038.

[26] M. Sagawa, S. Fujimura, N. Togawa, H. Yamamoto, Y. Matsuura, New material for permanent magnets on a base of Nd and Fe, J. Appl. Phys. 55 (6) (1984) 2083 http://dx.doi.org/10.1063/1.333572 (invited).

[27] B.D. Cullity, Introduction to Magnetic Materials, 2nd ed., IEEE/Wiley, Hoboken, NJ, 2009.

[28] L. Lagorce, O. Brand, M. Allen, Magnetic microactuators based on polymer magnets, J. Microelectromech. Syst. 8 (1) (1999) 2-9, http://dx.doi.org/10.1109/84. 749396.

[29] J. Streque, A. Talbi, C. Bonnerot, P. Pernod, V. Preobrazhensky, Magnetostatic micro-actuator based on ultrasoft elastomeric membrane and copper - permalloy electrodeposited structures, in: Proceedings of the IEEE International Conference on Micro Electro Mechanical Systems (MEMS), 2012, pp. 1157-1160.

\section{Biographies}

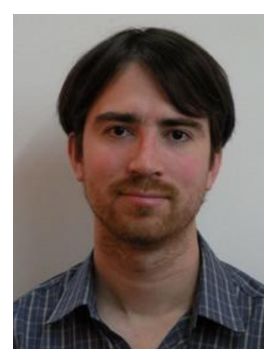

Juan José Zárate is a postdoctoral researcher at the EPFL in Switzerland. He received a M.Sc. in physics in 2007 and a Ph.D. in physics in 2013, both at Instituto Balseiro, Argentina. The doctoral research covered the development of an infrared bolometer sensor based on MEMS, as member of the Low Temperature Physics Laboratory, Centro Atómico Bariloche. In 2014, Juan joined the Microsystems for Space Technologies Laboratory (LMTS) for working in tactile displays. His research interests include polymer MEMS fabrication techniques and softmaterials sensors and actuators.

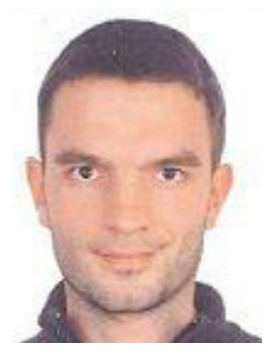

Giordano Tosolini is postdoctoral researcher at the Italian Institute of Technology. He received the Engineer's degree in materials engineering from the University of Trieste (Italy), in 2007, and Ph.D. in electronic engineering from Autonomous University of Barcelona (Spain), in 2013. His research interests are focused on piezoresistive and magnetic MEMS sensors and actuators.

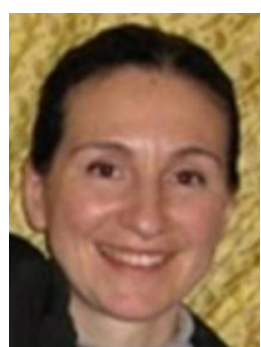

Simona Petroni received her M.S. degree in organic chemistry at University of Bari (Italy) in 1998. After a fellowship at INFM on MOCVD of II-VI semiconductors; in the 2000 she joined STMicroelectronics, working first as R\&D Process Engineer and then as MEMS Designer for the MEMS BU. In collaboration with National Nanotechnology Laboratory she developed AIN and GaN piezoelectric materials for surface acoustic waves (SAW) devices. In the 2007 she moved to Dublin to work for Hewlett-Packard on thermal MEMS. In the 2009 she came back to Italy for leading the research on soft MEMS applied to Robotics at CBN-IIT. 


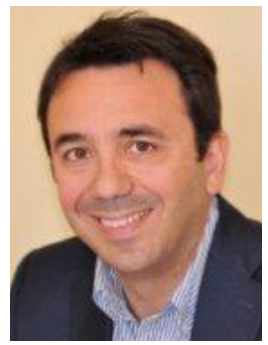

Massimo De Vittorio is director of the Center for Biomolecular Nanotechnologies (CBN) of the Istituto Italiano di Tecnologia (IIT), associate professor at Universitá del Salento and co-founder of the nanodevice division at the national nanotechnology laboratory (NNL) of the Istituto Nanoscienze CNR. His research activity deals with the development of science and technology applied to nanophotonics, nanoelectronics and nano and micro electromechanical systems (NEMS/MEMS). Author of about 240 papers and 13 patents, he is also senior editor of the IEEE Transactions on Nanotechnology.

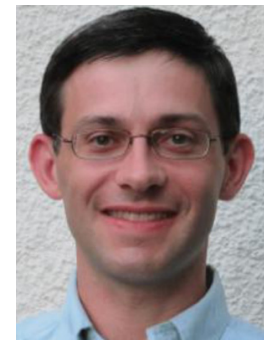

Herbert Shea is a professor at the EPFL in Switzerland, leading a team of a dozen scientists developing miniaturized elastomer-based actuators. After receiving a Ph.D. (1997) in physics at Harvard University, Herb spent 2 years a post-doctoral fellow at IBM's T.J. Watson Research Center, then joined Lucent Technologies' Bell Labs, becoming the technical manager of the Microsystems Technology group. In 2004 Herb founded the Microsystems for Space Technologies Laboratory (LMTS) at the EPFL. His current research topics include elastomer-based actuators, sensors, and energy harvesters, micromachined ion propulsion, and tactile displays. 